\title{
Crystal structure of chloro[1,2,3,4-(tetramethoxycarbonyl)buta-1,3-dien- 1,4-diyl]-bis(triphenylphosphine)iridium(III), $\left[\mathrm{Ir}\left\{\mathrm{C}_{4}\left(\mathrm{COOCH}_{3}\right)_{4}\right\} \mathrm{Cl}\left\{\mathbf{P}\left(\mathrm{C}_{6} \mathrm{H}_{5}\right)_{3}\right\}_{2}\right]$
}

\author{
M. Konkol, Ch. Wagner, C. Bruhn and D. Steinborn* \\ Martin-Luther-Universität Halle-Wittenberg, Institut für Anorganische Chemie, Kurt-Mothes-Str. 2, D-06120 Halle (Saale), Germany
}

Received December 4, 2002, accepted and available on-line January 23, 2003; CCDC-No. 1267/987

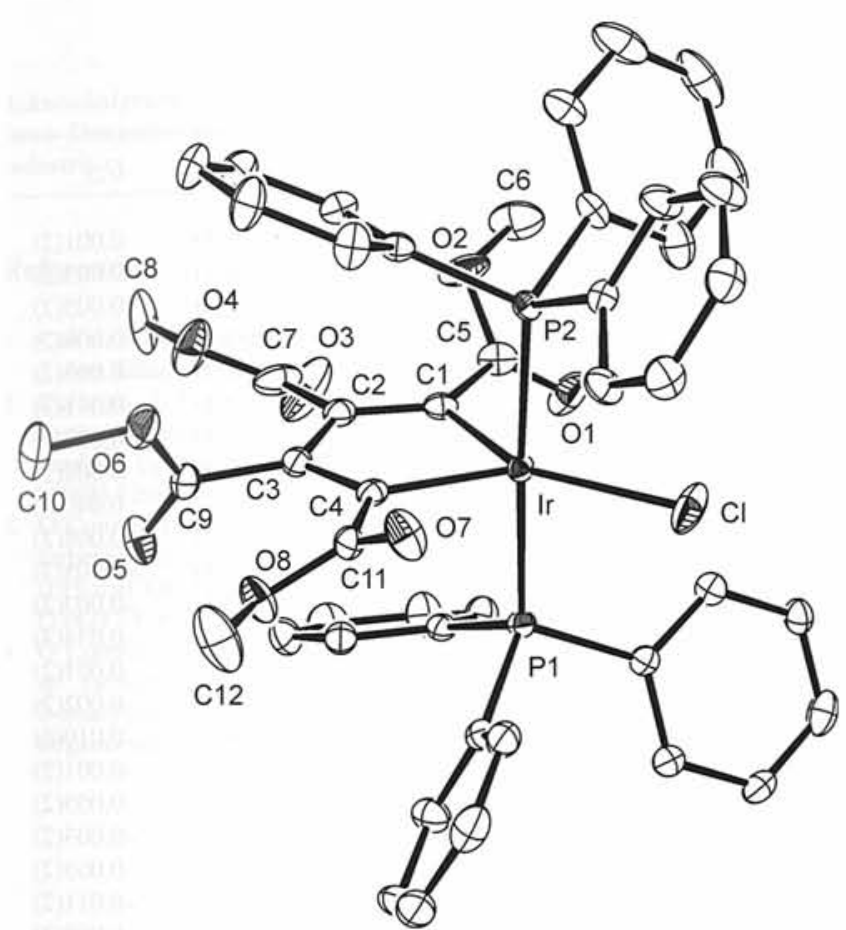

Abstract

$\mathrm{C}_{48} \mathrm{H}_{42} \mathrm{ClIrO}_{8} \mathrm{P}_{2}$, orthorhombic, $P 2{ }_{1} 2_{1} 2_{1}$ (No. 19),

$a=21.146(3) \AA, b=11.198(2) \AA, c=18.200(4) \AA, V=4309.6 \AA^{3}$, $Z=4, R_{\mathrm{gt}}(F)=0.021, w R_{\mathrm{ref}}\left(F^{2}\right)=0.040, T=220 \mathrm{~K}$.

\section{Source of material}

The reaction of $\left[\mathrm{IrCl}\left(\mathrm{N}_{2}\right)\left(\mathrm{PPh}_{3}\right)_{2}\right]$ with $(\mathrm{MeOOC})_{2} \mathrm{C} \equiv$ $\mathrm{C}(\mathrm{COOMe})_{2}$ in acetone at $283 \mathrm{~K}$ resulted in the formation of the title complex $\left[\mathrm{Ir}\left\{\mathrm{C}_{4}(\mathrm{COOMe})_{4}\right\} \mathrm{Cl}\left(\mathrm{PPh}_{3}\right)_{2}\right]$ which was previously obtained in the analogous reaction in thiophene-free benzene at $313 \mathrm{~K}-318 \mathrm{~K}[1,2]$. Crystals were obtained from methanol/chloroform solution.

\section{Experimental details}

Two carbon atoms $\mathrm{C} 8$ and $\mathrm{C} 8 \mathrm{~A}$ of the ester group on $\mathrm{C} 2$ are disordered over two positions with equal occupancies $(0.50)$. For clarity only one position is shown in the figure. The hydrogen atoms of that methyl group were not localized and not included into the model. All other hydrogen atoms are placed in calculated positions according to the riding model.

\footnotetext{
* Correspondence author (e-mail: steinborn@chemie.uni-halle.de)
}

\section{Discussion}

There are several examples of structurally characterized iridacyclopentadiene complexes [3,4]. However, five-fold coordination in mononuclear complexes is found only in one single case [5]. Here we report the second example of that type.

The title compound crystallizes as discrete molecules without any unusual intermolecular interactions. The trigonal bipyramidal co-ordination sphere of Ir is built up by two $\mathrm{PPh}_{3}$ ligands in apical positions. The angle P1-Ir-P2 is almost linear with $177.64(3)^{\circ}$. Equatorial ligands are the chloro ligand and the $\sigma$-bound buta-1,3-dien-1,4-diyl ligand. The sum of angles in the equatorial plane $\left(\angle \mathrm{C} 1-\mathrm{Ir}-\mathrm{C} 4=76.4(2)^{\circ}, \angle \mathrm{C} 1-\mathrm{Ir}-\mathrm{Cl}=146.8(1)^{\circ}\right.$, $\left.\angle \mathrm{C} 4-\mathrm{Ir}-\mathrm{Cl}=136.8(1)^{\circ}\right)$ amounts to $360.0^{\circ}$. The $\mathrm{Ir}-\mathrm{Cl}$ and $\mathrm{Ir}-\mathrm{C} 4$ bond distances are 2.015(4) $\AA$ and 2.011(4) $\AA$, respectively. Overall, there are no remarkable differences in the bond lengths and angles of the title complex compared with those in other iridacyclopentadiene complexes.

Table 1. Data collection and handling.

Crystal:

Wavelength:

$\mu$ :

Diffractometer, scan mode:

$2 \theta_{\max }$ :

$N(h k l)_{\text {measured }}, N(h k l)_{\text {unique: }}$

Criterion for $I_{\mathrm{obs}}, N(h k l)_{\mathrm{gt}}$ :

$N(\text { param })_{\text {refined: }}$

Programs:

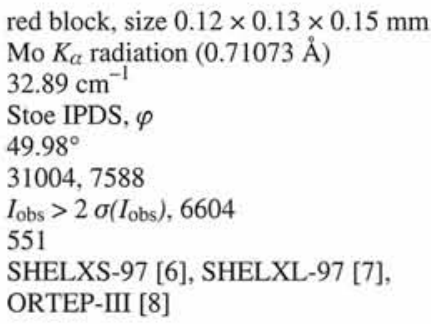

Table 2. Atomic coordinates and displacement parameters (in $\AA^{2}$ ).

\begin{tabular}{llllll}
\hline Atom & Site & $x$ & $y$ & $U_{\text {iso }}$ \\
\hline $\mathrm{H}(3)$ & $4 a$ & 0.7338 & 1.1813 & 0.4224 & 0.104 \\
$\mathrm{H}(2)$ & $4 a$ & 0.6746 & 1.1950 & 0.3713 & 0.104 \\
$\mathrm{H}(1)$ & $4 a$ & 0.7391 & 1.2534 & 0.3488 & 0.104 \\
$\mathrm{H}(4)$ & $4 a$ & 0.8569 & 0.7391 & 0.0154 & 0.103 \\
$\mathrm{H}(6)$ & $4 a$ & 0.8097 & 0.6314 & 0.0098 & 0.103 \\
$\mathrm{H}(5)$ & $4 a$ & 0.8746 & 0.6156 & 0.0504 & 0.103 \\
$\mathrm{H}(9)$ & $4 a$ & 0.8229 & 0.3430 & 0.2147 & 0.112 \\
$\mathrm{H}(8)$ & $4 a$ & 0.8656 & 0.3835 & 0.1489 & 0.112 \\
$\mathrm{H}(7)$ & $4 a$ & 0.7922 & 0.4022 & 0.1453 & 0.112 \\
$\mathrm{H}(14)$ & $4 a$ & 0.9247 & 0.6003 & 0.5510 & 0.036 \\
$\mathrm{H}(15)$ & $4 a$ & 0.9237 & 0.6162 & 0.6793 & 0.043 \\
$\mathrm{H}(16)$ & $4 a$ & 0.8507 & 0.7415 & 0.7353 & 0.053 \\
$\mathrm{H}(17)$ & $4 a$ & 0.7819 & 0.8531 & 0.6664 & 0.042 \\
$\mathrm{H}(18)$ & $4 a$ & 0.7806 & 0.8339 & 0.5399 & 0.037 \\
$\mathrm{H}(20)$ & $4 a$ & 0.9805 & 0.6080 & 0.4151 & 0.047 \\
$\mathrm{H}(21)$ & $4 a$ & 1.0239 & 0.4240 & 0.3891 & 0.057 \\
& & & & & \\
\hline
\end{tabular}


Table 2. Continued.

\begin{tabular}{llllll}
\hline Atom & Site & $x$ & $y$ & $z$ & $U_{\text {iso }}$ \\
\hline $\mathrm{H}(22)$ & $4 a$ & 0.9593 & 0.2611 & 0.3719 & 0.062 \\
$\mathrm{H}(23)$ & $4 a$ & 0.8514 & 0.2793 & 0.3836 & 0.058 \\
$\mathrm{H}(24)$ & $4 a$ & 0.8068 & 0.4649 & 0.4084 & 0.044 \\
$\mathrm{H}(26)$ & $4 a$ & 0.9078 & 0.9181 & 0.4930 & 0.048 \\
$\mathrm{H}(27)$ & $4 a$ & 0.9820 & 1.0567 & 0.4559 & 0.060 \\
$\mathrm{H}(28)$ & $4 a$ & 1.0306 & 1.0404 & 0.3424 & 0.064 \\
$\mathrm{H}(29)$ & $4 a$ & 1.0077 & 0.8793 & 0.2678 & 0.054 \\
$\mathrm{H}(30)$ & $4 a$ & 0.9347 & 0.7376 & 0.3048 & 0.041 \\
$\mathrm{H}(32)$ & $4 a$ & 0.5662 & 0.9319 & 0.2667 & 0.054 \\
$\mathrm{H}(33)$ & $4 a$ & 0.5145 & 1.0936 & 0.3198 & 0.079 \\
$\mathrm{H}(34)$ & $4 a$ & 0.5269 & 1.1363 & 0.4417 & 0.086 \\
$\mathrm{H}(35)$ & $4 a$ & 0.5959 & 1.0257 & 0.5114 & 0.092 \\
& & & & &
\end{tabular}

Table 2. Continued.

\begin{tabular}{llllll}
\hline Atom & Site & $x$ & $y$ & $z$ & $U_{\text {iso }}$ \\
\hline $\mathrm{H}(36)$ & $4 a$ & 0.6467 & 0.8630 & 0.4622 & 0.063 \\
$\mathrm{H}(38)$ & $4 a$ & 0.5178 & 0.7297 & 0.3462 & 0.047 \\
$\mathrm{H}(39)$ & $4 a$ & 0.4456 & 0.5788 & 0.3672 & 0.057 \\
$\mathrm{H}(40)$ & $4 a$ & 0.4786 & 0.3821 & 0.3758 & 0.047 \\
$\mathrm{H}(41)$ & $4 a$ & 0.5838 & 0.3375 & 0.3630 & 0.047 \\
$\mathrm{H}(42)$ & $4 a$ & 0.6577 & 0.4871 & 0.3423 & 0.041 \\
$\mathrm{H}(44)$ & $4 a$ & 0.6815 & 0.9337 & 0.2192 & 0.039 \\
$\mathrm{H}(45)$ & $4 a$ & 0.6856 & 0.9514 & 0.0929 & 0.043 \\
$\mathrm{H}(46)$ & $4 a$ & 0.6568 & 0.7942 & 0.0195 & 0.052 \\
$\mathrm{H}(47)$ & $4 a$ & 0.6239 & 0.6171 & 0.0713 & 0.059 \\
$\mathrm{H}(48)$ & $4 a$ & 0.6221 & 0.5946 & 0.1979 & 0.045 \\
& & & & & \\
\hline
\end{tabular}

Table 3. Atomic coordinates and displacement parameters (in $\AA^{2}$ ).

\begin{tabular}{|c|c|c|c|c|c|c|c|c|c|c|c|}
\hline Atom & Site & Occ. & $x$ & $y$ & $z$ & $U_{11}$ & $U_{22}$ & $U_{33}$ & $U_{12}$ & $U_{13}$ & $U_{23}$ \\
\hline$C(1)$ & $4 a$ & & $0.7805(2)$ & $0.8834(3)$ & $0.3311(2)$ & $0.021(2)$ & $0.026(2)$ & $0.034(2)$ & $-0.001(2)$ & $-0.006(2)$ & $0.001(2)$ \\
\hline $\mathrm{C}(2)$ & $4 a$ & & $0.8114(2)$ & $0.8859(4)$ & $0.2656(2)$ & $0.023(2)$ & $0.034(2)$ & $0.034(2)$ & $0.001(2)$ & $-0.004(2)$ & $0.005(2)$ \\
\hline $\mathrm{C}(3)$ & $4 a$ & & $0.8173(2)$ & $0.7656(4)$ & $0.2328(2)$ & $0.021(2)$ & $0.038(2)$ & $0.027(2)$ & $-0.001(2)$ & $-0.001(2)$ & $0.005(2)$ \\
\hline$C(4)$ & $4 a$ & & $0.7918(2)$ & $0.6803(3)$ & $0.2771(2)$ & $0.018(2)$ & $0.029(2)$ & $0.028(2)$ & $-0.002(2)$ & $-0.004(2)$ & $0.006(2)$ \\
\hline$C(5)$ & $4 a$ & & $0.7662(2)$ & $0.9933(3)$ & $0.3743(4)$ & $0.036(3)$ & $0.025(2)$ & $0.048(2)$ & $-0.004(1)$ & $-0.008(3)$ & $-0.003(2)$ \\
\hline $\mathrm{C}(6)$ & $4 a$ & & $0.7197(3)$ & $1.1860(4)$ & $0.3723(4)$ & $0.083(3)$ & $0.039(2)$ & $0.087(4)$ & $0.017(2)$ & $-0.022(4)$ & $-0.011(3)$ \\
\hline$C(7)$ & $4 a$ & & $0.8378(3)$ & $0.9921(4)$ & $0.2296(4)$ & $0.039(3)$ & $0.044(3)$ & $0.081(5)$ & $-0.003(2)$ & $-0.008(3)$ & $0.037(3)$ \\
\hline $\mathrm{C}(8 \mathrm{~A})$ & $4 a$ & $0.50(1)$ & $0.9085(5)$ & $1.1471(8)$ & $0.2229(6)$ & $0.074(8)$ & $0.036(6)$ & $0.069(8)$ & $-0.027(5)$ & $0.025(6)$ & $0.002(5)$ \\
\hline$C(8)$ & $4 a$ & 0.50 & $0.8679(8)$ & $1.1114(8)$ & $0.1328(7)$ & $0.18(2)$ & $0.035(6)$ & $0.08(1)$ & $0.031(7)$ & $0.10(1)$ & $0.040(6)$ \\
\hline $\mathrm{C}(9)$ & $4 a$ & & $0.8503(2)$ & $0.7428(4)$ & $0.1616(2)$ & $0.035(2)$ & $0.038(3)$ & $0.029(2)$ & $-0.002(2)$ & $0.006(2)$ & $0.008(2)$ \\
\hline$C(10)$ & $4 a$ & & $0.8406(3)$ & $0.6700(6)$ & $0.0403(3)$ & $0.095(4)$ & $0.079(4)$ & $0.031(3)$ & $-0.003(4)$ & $0.014(3)$ & $-0.005(3)$ \\
\hline$C(11)$ & $4 a$ & & $0.7879(2)$ & $0.5517(4)$ & $0.2544(2)$ & $0.030(2)$ & $0.033(2)$ & $0.022(2)$ & $0.001(2)$ & $-0.000(2)$ & $-0.003(2)$ \\
\hline$C(12)$ & $4 a$ & & $0.8285(3)$ & $0.4020(5)$ & $0.1770(4)$ & $0.083(4)$ & $0.048(3)$ & $0.093(5)$ & $-0.014(3)$ & $0.039(4)$ & $-0.034(3)$ \\
\hline$C(13)$ & $4 a$ & & $0.8526(2)$ & $0.7154(3)$ & $0.5325(2)$ & $0.029(2)$ & $0.020(2)$ & $0.028(2)$ & $-0.003(2)$ & $0.000(2)$ & $0.001(2)$ \\
\hline$C(14)$ & $4 a$ & & $0.8957(2)$ & $0.6501(3)$ & $0.5741(2)$ & $0.028(2)$ & $0.029(2)$ & $0.033(2)$ & $-0.004(2)$ & $-0.002(2)$ & $0.002(2)$ \\
\hline$C(15)$ & $4 a$ & & $0.8952(2)$ & $0.6598(4)$ & $0.6510(2)$ & $0.037(2)$ & $0.038(3)$ & $0.032(2)$ & $-0.004(2)$ & $-0.007(2)$ & $0.010(2)$ \\
\hline$C(16)$ & $4 a$ & & $0.8516(2)$ & $0.7355(4)$ & $0.6843(2)$ & $0.055(3)$ & $0.052(3)$ & $0.026(2)$ & $-0.019(3)$ & $-0.002(2)$ & $-0.001(2)$ \\
\hline$C(17)$ & $4 a$ & & $0.8100(2)$ & $0.8012(4)$ & $0.6433(2)$ & $0.041(2)$ & $0.035(2)$ & $0.029(2)$ & $0.001(2)$ & $0.006(2)$ & $-0.005(2)$ \\
\hline$C(18)$ & $4 a$ & & $0.8096(2)$ & $0.7906(3)$ & $0.5675(2)$ & $0.034(2)$ & $0.028(2)$ & $0.031(2)$ & $-0.002(2)$ & $-0.001(2)$ & $-0.003(2)$ \\
\hline$C(19)$ & $4 a$ & & $0.8896(2)$ & $0.5579(4)$ & $0.4125(2)$ & $0.038(2)$ & $0.027(2)$ & $0.022(2)$ & $0.005(2)$ & $-0.003(2)$ & $-0.003(2)$ \\
\hline$C(20)$ & $4 a$ & & $0.9540(2)$ & $0.5427(4)$ & $0.4079(3)$ & $0.039(3)$ & $0.040(3)$ & $0.040(3)$ & $0.007(2)$ & $-0.006(2)$ & $-0.011(2)$ \\
\hline$C(21)$ & $4 a$ & & $0.9803(2)$ & $0.4323(5)$ & $0.3927(3)$ & $0.051(3)$ & $0.050(3)$ & $0.041(3)$ & $0.026(3)$ & $-0.004(2)$ & $-0.008(2)$ \\
\hline $\mathrm{C}(22)$ & $4 a$ & & $0.9417(2)$ & $0.3351(4)$ & $0.3829(3)$ & $0.074(3)$ & $0.041(3)$ & $0.041(3)$ & $0.025(3)$ & $0.007(3)$ & $-0.001(2)$ \\
\hline $\mathrm{C}(23)$ & $4 a$ & & $0.8772(3)$ & $0.3460(4)$ & $0.3892(2)$ & $0.082(3)$ & $0.030(2)$ & $0.034(3)$ & $-0.005(2)$ & $0.000(2)$ & $-0.001(2)$ \\
\hline$C(24)$ & $4 a$ & & $0.8504(2)$ & $0.4573(4)$ & $0.4041(2)$ & $0.044(3)$ & $0.034(2)$ & $0.032(2)$ & $0.003(2)$ & $0.002(2)$ & $0.002(2)$ \\
\hline$C(25)$ & $4 a$ & & $0.9140(2)$ & $0.8130(3)$ & $0.4029(2)$ & $0.020(2)$ & $0.034(2)$ & $0.030(2)$ & $0.001(2)$ & $-0.002(2)$ & $0.004(2)$ \\
\hline$C(26)$ & $4 a$ & & $0.9280(2)$ & $0.9096(4)$ & $0.4479(3)$ & $0.042(3)$ & $0.038(3)$ & $0.041(3)$ & $-0.007(2)$ & $0.003(2)$ & $0.003(2)$ \\
\hline $\mathrm{C}(27)$ & $4 a$ & & $0.9721(3)$ & $0.9929(4)$ & $0.4253(3)$ & $0.051(4)$ & $0.047(3)$ & $0.052(4)$ & $-0.019(2)$ & $0.008(3)$ & $-0.005(2)$ \\
\hline $\mathrm{C}(28)$ & $4 a$ & & $1.0016(3)$ & $0.9830(4)$ & $0.3576(3)$ & $0.043(3)$ & $0.049(3)$ & $0.069(5)$ & $-0.019(2)$ & $0.005(3)$ & $0.011(3)$ \\
\hline $\mathrm{C}(29)$ & $4 a$ & & $0.9877(2)$ & $0.8871(4)$ & $0.3131(3)$ & $0.032(2)$ & $0.060(3)$ & $0.044(3)$ & $-0.001(2)$ & $0.008(2)$ & $0.003(2)$ \\
\hline$C(30)$ & $4 a$ & & $0.9440(2)$ & $0.8021(4)$ & $0.3352(2)$ & $0.024(2)$ & $0.048(3)$ & $0.031(2)$ & $-0.002(2)$ & $-0.004(2)$ & $-0.000(2)$ \\
\hline $\mathrm{C}(31)$ & $4 a$ & & $0.6116(2)$ & $0.8790(3)$ & $0.3581(2)$ & $0.026(2)$ & $0.026(2)$ & $0.042(3)$ & $-0.002(2)$ & $0.010(2)$ & $-0.007(2)$ \\
\hline $\mathrm{C}(32)$ & $4 a$ & & $0.5722(2)$ & $0.9490(4)$ & $0.3162(3)$ & $0.035(3)$ & $0.037(3)$ & $0.062(3)$ & $0.006(2)$ & $0.006(2)$ & $-0.004(2)$ \\
\hline $\mathrm{C}(33)$ & $4 a$ & & $0.5410(3)$ & $1.0464(5)$ & $0.3484(4)$ & $0.045(3)$ & $0.030(3)$ & $0.122(6)$ & $0.011(2)$ & $0.015(3)$ & $0.002(3)$ \\
\hline $\mathrm{C}(34)$ & $4 a$ & & $0.5489(3)$ & $1.0729(5)$ & $0.4207(4)$ & $0.067(4)$ & $0.040(3)$ & $0.108(6)$ & $0.001(3)$ & $0.047(4)$ & $-0.028(4)$ \\
\hline$C(35)$ & $4 a$ & & $0.5893(4)$ & $1.0057(5)$ & $0.4625(4)$ & $0.092(5)$ & $0.067(5)$ & $0.071(5)$ & $-0.006(3)$ & $0.034(4)$ & $-0.032(3)$ \\
\hline$C(36)$ & $4 a$ & & $0.6201(3)$ & $0.9089(4)$ & $0.4329(3)$ & $0.063(3)$ & $0.051(3)$ & $0.045(3)$ & $-0.003(3)$ & $0.016(3)$ & $-0.015(2)$ \\
\hline$C(37)$ & $4 a$ & & $0.5952(2)$ & $0.6255(3)$ & $0.3415(2)$ & $0.027(2)$ & $0.026(2)$ & $0.023(2)$ & $-0.006(2)$ & $-0.002(2)$ & $-0.001(2)$ \\
\hline$C(38)$ & $4 a$ & & $0.5316(2)$ & $0.6510(4)$ & $0.3494(3)$ & $0.026(2)$ & $0.034(2)$ & $0.058(3)$ & $-0.000(2)$ & $0.004(2)$ & $0.008(2)$ \\
\hline$C(39)$ & $4 a$ & & $0.4883(2)$ & $0.5604(4)$ & $0.3621(3)$ & $0.024(2)$ & $0.047(3)$ & $0.070(4)$ & $0.001(2)$ & $0.005(2)$ & $0.003(3)$ \\
\hline $\mathrm{C}(40)$ & $4 a$ & & $0.5079(2)$ & $0.4426(4)$ & $0.3673(3)$ & $0.037(2)$ & $0.040(3)$ & $0.041(3)$ & $-0.013(2)$ & $0.002(2)$ & $0.002(2)$ \\
\hline$C(41)$ & $4 a$ & & $0.5705(2)$ & $0.4164(4)$ & $0.3597(2)$ & $0.044(2)$ & $0.028(2)$ & $0.046(3)$ & $-0.005(2)$ & $0.005(2)$ & $0.003(2)$ \\
\hline$C(42)$ & $4 a$ & & $0.6151(2)$ & $0.5062(3)$ & $0.3470(3)$ & $0.022(2)$ & $0.034(3)$ & $0.046(3)$ & $-0.005(2)$ & $0.005(2)$ & $0.001(2)$ \\
\hline$C(43)$ & $4 a$ & & $0.6511(2)$ & $0.7626(4)$ & $0.2227(2)$ & $0.023(2)$ & $0.025(2)$ & $0.032(2)$ & $0.000(2)$ & $-0.004(2)$ & $-0.001(2)$ \\
\hline$C(44)$ & $4 a$ & & $0.6702(2)$ & $0.8691(4)$ & $0.1899(2)$ & $0.033(2)$ & $0.029(2)$ & $0.036(2)$ & $-0.002(2)$ & $-0.008(2)$ & $0.004(2)$ \\
\hline $\mathrm{C}(45)$ & $4 a$ & & $0.6724(2)$ & $0.8800(3)$ & $0.1141(2)$ & $0.043(2)$ & $0.028(2)$ & $0.037(3)$ & $-0.004(2)$ & $0.000(2)$ & $0.008(2)$ \\
\hline$C(46)$ & $4 a$ & & $0.6553(2)$ & $0.7863(4)$ & $0.0703(2)$ & $0.056(3)$ & $0.050(3)$ & $0.025(2)$ & $-0.008(3)$ & $0.002(2)$ & $0.010(2)$ \\
\hline$C(47)$ & $4 a$ & & $0.6360(3)$ & $0.6803(4)$ & $0.1014(2)$ & $0.073(3)$ & $0.048(3)$ & $0.026(2)$ & $-0.014(3)$ & $0.001(2)$ & $-0.007(2)$ \\
\hline $\mathrm{C}(48)$ & $4 a$ & & $0.6343(2)$ & $0.6670(4)$ & $0.1773(2)$ & $0.051(3)$ & $0.028(2)$ & $0.035(2)$ & $-0.013(2)$ & $0.004(2)$ & $-0.004(2)$ \\
\hline $\mathrm{O}(1)$ & $4 a$ & & $0.7752(2)$ & $1.0030(2)$ & $0.4392(2)$ & $0.107(4)$ & $0.041(2)$ & $0.031(2)$ & $0.016(2)$ & $-0.007(2)$ & $-0.002(1)$ \\
\hline
\end{tabular}




\begin{tabular}{|c|c|c|c|c|c|c|c|c|c|c|}
\hline Atom & Site Occ. & $x$ & $y$ & $z$ & $U_{11}$ & $U_{22}$ & $U_{33}$ & $U_{12}$ & $U_{13}$ & $U_{23}$ \\
\hline $\mathrm{O}(2)$ & $4 a$ & $0.7376(2)$ & $1.0761(2)$ & $0.3333(2)$ & $0.083(3)$ & $0.025(1)$ & $0.056(2)$ & $0.019(2)$ & $-0.032(2)$ & $-0.004(1)$ \\
\hline$O(3)$ & $4 a$ & $0.8622(2)$ & $1.0726(4)$ & $0.2671(2)$ & $0.120(4)$ & $0.092(3)$ & $0.061(3)$ & $-0.072(3)$ & $-0.026(3)$ & $0.033(2)$ \\
\hline $\mathrm{O}(4)$ & $4 a$ & $0.8380(3)$ & $0.9966(3)$ & $0.1591(2)$ & $0.121(4)$ & $0.048(2)$ & $0.045(2)$ & $-0.012(2)$ & $0.011(3)$ & $0.018(2)$ \\
\hline $\mathrm{O}(5)$ & $4 a$ & $0.9065(1)$ & $0.7541(3)$ & $0.1530(2)$ & $0.043(2)$ & $0.061(2)$ & $0.049(2)$ & $-0.011(2)$ & $0.019(1)$ & $-0.009(2)$ \\
\hline $\mathrm{O}(6)$ & $4 a$ & $0.8111(1)$ & $0.7064(3)$ & $0.1091(2)$ & $0.053(2)$ & $0.071(2)$ & $0.029(2)$ & $-0.000(2)$ & $-0.004(1)$ & $-0.003(2)$ \\
\hline$O(7)$ & $4 a$ & $0.7467(2)$ & $0.4837(2)$ & $0.2731(2)$ & $0.043(2)$ & $0.040(1)$ & $0.062(2)$ & $-0.014(2)$ & $0.018(2)$ & $-0.011(1)$ \\
\hline $\mathrm{O}(8)$ & $4 a$ & $0.8357(2)$ & $0.5194(3)$ & $0.2106(2)$ & $0.042(2)$ & $0.033(2)$ & $0.052(2)$ & $-0.005(1)$ & $0.018(2)$ & $-0.015(1)$ \\
\hline$P(1)$ & $4 a$ & $0.85349(4)$ & $0.70412(8)$ & $0.43100(5)$ & $0.0234(4)$ & $0.0242(6)$ & $0.0243(5)$ & $0.0015(4)$ & $-0.0016(4)$ & $0.0002(4)$ \\
\hline $\mathrm{P}(2)$ & $4 a$ & $0.65224(4)$ & $0.74551(8)$ & $0.32268(5)$ & $0.0206(4)$ & $0.0237(6)$ & $0.0261(5)$ & $-0.0019(4)$ & $0.0016(4)$ & $-0.0011(4)$ \\
\hline $\mathrm{Cl}$ & $4 a$ & $0.70776(6)$ & $0.6249(1)$ & $0.47583(6)$ & $0.0468(7)$ & $0.091(1)$ & $0.0357(6)$ & $-0.0316(7)$ & $-0.0073(6)$ & $0.0283(6)$ \\
\hline Ir & $4 a$ & $0.753669(7)$ & $0.72575(1)$ & $0.374405(7)$ & $0.02115(6)$ & $0.02164(5)$ & $0.02123(5)$ & $-0.00184(7)$ & $0.00020(8)$ & $0.00180(6)$ \\
\hline
\end{tabular}

Acknowledgments. The authors gratefully acknowledge the financial support from Deutsche Forschungsgemeinschaft and Fonds der Chemischen Industrie.

\section{References}

1. Collman, J.P.; Kang, J. W.: Acetylene complexes of iridium and rhodium. J. Am. Chem. Soc. 89 (1967) 844-851.

2. Collman, J. P.; Kang, J. W.; Little, W. F.; Sullivan, M. F.: Metallocyclopentadiene complexes of iridium and rhodium and their role in the catalytic cyclotrimerization of disubstituted acetylenes. Inorg. Chem. 7 (1968) 1298-1303.

3. O'Connor, J. M.; Pu, L.; Rheingold, A. L.: The first stable metallacyclecarbene complexes: structural characterization of [cyclic] $\operatorname{Ir}(\mathrm{CR}: \mathrm{CRCR}: \mathrm{CR})$ $\left(\mathrm{PPh}_{3}\right)_{2}(\mathrm{CO})\left(: \mathrm{C}\left(\mathrm{CH}_{2}\right)_{3} \mathrm{O}\right)^{+} \mathrm{BF}_{4}^{-}, \mathrm{R}=\mathrm{CO}_{2} \mathrm{CH}_{3}$. J. Am. Chem. Soc. 109 (1987) 7578-7579.

4. O'Connor, J. M.; Pu, L.; Rheingold, A. L.: Nucleophilic cleavage of the $s p^{3}$ carbon-oxygen bond in alkoxycarbene complexes: conversion of 2-oxacyclopentylidene ligands to pyridinium-substituted acyl ligands. Organometallics 7 (1988) 2060-2062.
5. Suenkel, K.: Coordination chemistry of perhalogenated cyclopentadienes and alkynes. VII. Synthesis of several tetrachlorometalloles of cobalt, rhodium and iridium; structure of an iridacyclopentadiene derivative. Chem. Ber. 124 (1991) 2449-2451.

6. Sheldrick, G. M.: SHELXS-97. Program for the Solution of Crystal Structures. University of Göttingen, Germany 1997.

7. Sheldrick, G. M.: SHELXL-97. Program for the Refinement of Crystal Structures. University of Göttingen, Germany 1997.

8. Johnson, C. K.; Burnett, M. N.: ORTEP-III version 1.0.2.: Oak Ridge Thermal Ellipsoid Plot Program for Crystal Structure Illustrations, Oak Ridge National Laboratory Report ORNL-6895, USA 1996; Farrugia, L. J.: Windows 32-bit version 1.04, University of Glasgow, UK 1998. 